\title{
Self-reported nonadherence to antiretroviral therapy as a predictor of viral failure and mortality
}

\author{
Tracy R. Glass ${ }^{a, b, c}$, Jonathan A.C. Sterne ${ }^{d}$, Marie-Paule Schneider ${ }^{e, f}$, \\ Sabina De Geest ${ }^{\mathrm{g}}$, Dunja Niccah ${ }^{\text {, }}$ Hansjakob Furreri, \\ Huldrych F. Günthardi, Enos Bernasconik, Alexandra Calmy', \\ Martin Rickenbach ${ }^{\mathrm{m}}$, Manuel Battegay ${ }^{\mathrm{n}}$, Heiner C. Bucher ${ }^{\mathrm{a}, \mathrm{n}}$, \\ the Swiss HIV Cohort Study
}

\begin{abstract}
Objective: To determine the effect of nonadherence to antiretroviral therapy (ART) on virologic failure and mortality in naive individuals starting ART.

Design: Prospective observational cohort study.

Methods: Eligible individuals enrolled in the Swiss HIV Cohort Study, started ART between 2003 and 2012, and provided adherence data on at least one biannual clinical visit. Adherence was defined as missed doses (none, one, two, or more than two) and percentage adherence $(>95,90-95$, and $<90)$ in the previous 4 weeks. Inverse probability weighting of marginal structural models was used to estimate the effect of nonadherence on viral failure (HIV-1 viral load $>500$ copies $/ \mathrm{ml}$ ) and mortality.

Results: Of 3150 individuals followed for a median 4.7 years, 480 (15.2\%) experienced viral failure and $104(3.3 \%)$ died, $1155(36.6 \%)$ reported missing one dose, $414(13.1 \%)$ two doses and, $333(10.6 \%)$ more than two doses of ART. The risk of viral failure increased with each missed dose (one dose: hazard ratio [HR] 1.15, 95\% confidence interval 0.79-1.67; two doses: 2.15, 1.31-3.53; more than two doses: 5.21, 2.969.18). The risk of death increased with more than two missed doses (HR 4.87, 2.21 10.73). Missing one to two doses of ART increased the risk of viral failure in those starting once-daily (HR 1.67, 1.11-2.50) compared with those starting twice-daily regimens (HR 0.99, 0.64-1.54, interaction $P=0.09$ ). Consistent results were found for percentage adherence.
\end{abstract}

\footnotetext{
${ }^{a}$ Basel Institute for Clinical Epidemiology and Biostatistics, University Hospital Basel, ${ }^{b}$ Swiss Tropical and Public Health Institute, ${ }^{\mathrm{C}}$ University of Basel, Basel, Switzerland, ${ }^{\mathrm{d} D e p a r t m e n t}$ of Social Medicine, University of Bristol, Bristol, UK, ${ }^{\mathrm{e} C o m m u n i t y}$ pharmacy, Department of Ambulatory Care and Community Medicine, University of Lausanne, ${ }^{f}$ Community pharmacy, School of Pharmaceutical Sciences, University of Geneva, University of Lausanne, Lausanne, gInstitute of Nursing Science, University of Basel, Basel, hDivision of Infectious Diseases, Kantonsspital St. Gallen, St. Gallen, 'Department of Infectious Diseases, Bern University Hospital and University of Bern, Bern, 'Division of Infectious Diseases and Hospital Epidemiology University Hospital Zurich and University of Zurich, Zurich, ${ }^{\mathrm{k}}$ Division of Infectious Diseases, Ospedale Civico Lugano, Lugano, Division of Infectious Diseases, University Hospital Geneva, Geneva, ${ }^{\mathrm{m}}$ Swiss HIV Cohort Data Center, University Institute of Social and Preventive Medicine Lausanne, Lausanne, and ${ }^{\mathrm{n}}$ Division of Infectious Diseases and Hospital Epidemiology, University Hospital Basel, Basel, Switzerland.

Correspondence to Tracy R. Glass, Swiss Tropical and Public Health Institute, Socinstrasse 57, CH - 4051 Basel, Switzerland. Tel: +41 61284 8714; e-mail: Tracy.Glass@unibas.ch

Received: 13 March 2015; revised: 9 June 2015; accepted: 16 June 2015.
}

DOI:10.1097/QAD.0000000000000782

ISSN 0269-9370 Copyright @ 2015 Wolters Kluwer Health, Inc. All rights reserved. Copyright @ 2015 Wolters Kluwer Health, Inc. All rights reserved. 
Conclusion: Self-report of two or more missed doses of ART is associated with an increased risk of both viral failure and death. A simple adherence question helps identify patients at risk for negative clinical outcomes and offers opportunities for intervention.

Copyright @ 2015 Wolters Kluwer Health, Inc. All rights reserved.

AIDS 2015, 29:2195-2200

\section{Keywords: adherence, antiretroviral therapy, causal modeling, mortality, viral failure}

\section{Introduction}

Once started, antiretroviral therapy (ART) is lifelong with the aims of reducing HIV-related morbidity and mortality [1-3]. Prevention of nonadherence is of utmost importance for HIV disease management because nonadherence increases the risk of virological failure and thus of HIV transmission [4-7]. The relationship between nonadherence and progression to AIDS or death has rarely been investigated, and all but one study [6] used only standard statistical models to adjust for confounding [8-10]. Such standard approaches may produce biased effect estimates because they do not properly adjust for time-dependent confounding, which occurs when confounders, predictors of both exposure and outcome, are also affected by prior exposure. For example, choice of ART drug class may be related both to (expected) adherence and mortality risk, and prior adherence may influence future choice of ART drug class [1,11-13]. We estimated the relationship between nonadherence and clinical outcomes among antiretroviral-naive patients starting ART in the Swiss HIV Cohort Study (SHCS), using inverse probability weighting of marginal structural models to adjust for time-dependent confounding.

\section{Methods}

All antiretroviral-naive SHCS participants with prospectively collected data, who initiated ART between 1 January, 2003, and 1 January, 2012, and completed at least one SHCS adherence questionnaire (SHCS-AQ) during follow-up were included $[4,14,15]$. Outcomes were viral failure, defined as the first HIV-1 RNA viral load more than 500 copies $/ \mathrm{ml}$ after either achieving viral suppression (viral load $<50$ copies $/ \mathrm{ml}$ ) or being on therapy for longer than 24 weeks, and all-cause mortality. We also considered a cutoff for viral failure of viral load more than 50 copies $/ \mathrm{ml}$. The SHCS-AQ asks participants how often they missed a dose of ART in the previous 4 weeks (none, once a month, once every 2 weeks, once a week, more than once a week, and every day). These response categories were converted to missing none, one, two, or more than two doses of ART in the last 4 weeks, a more clinically meaningful definition that has been shown to be associated with viral failure and development of resistance mutations [4,7]. For comparability across regimens with different dosing frequencies, percentage adherence was also calculated and categorized as more than $95 \%, 90-95 \%$, and $90 \%$ or less.

Follow-up started at the date of ART initiation (baseline). The baseline confounders of the relationship between nonadherence and clinical outcomes included in the model are provided in Table 1. Confounders with time updated information were $\mathrm{CD} 4^{+}$cell count, viral load (mortality model only), time virally suppressed (viral failure model only), ART class, and dosing frequency. Follow-up continued until the outcome was observed, the individual was lost to follow-up ( $>12$ months since their last visit), or 1 December, 2013, whichever came first. As we are most interested in the total effect of nonadherence, individuals were not censored for intermediate endpoints, such as treatment discontinuation or switching. Follow-up of participants missing any time-dependent confounder at baseline started at the first subsequent visit at which the variable was observed.

Both unweighted and weighted (marginal structural models [MSMs]) pooled logistic regression models were developed for viral failure and mortality. In the MSM models, the analysis is weighted to create a pseudopopulation in which time-dependent confounders (which lie on the causal pathway) at time $t-1$ no longer predict nonadherence at time $t$ allowing for measurement of the total effect of exposure. Provided that there is no unmeasured confounding, the parameter of the MSM estimates the causal effect of nonadherence on viral failure or mortality (for details see eMethods) [11]. In sensitivity analyses, we explored replacing nonadherence as the exposure with the interaction between dosing frequency and nonadherence (missing no doses, missing one to two doses on a once-daily [q.d.] regimen, missing one to two doses on a twice-daily [b.i.d.] regimen, missing more than two doses on a q.d. regimen, and missing more than two doses on a b.i.d. regimen) [4].

\section{Results}

Of 3247 treatment-naive individuals initiating ART during the study period, $86(2.6 \%)$ never completed the SHCS-AQ, three $(0.1 \%)$ had HIV-2 virus, and three $(0.1 \%)$ did not have follow-up information. The final 
Table 1. Baseline demographic and clinical characteristics of participants.

\begin{tabular}{|c|c|c|c|}
\hline Variable & Overall & Adherent $^{\mathrm{a}}$ & Nonadherent $^{\mathrm{a}}$ \\
\hline N & 3155 & $1781(56.5)$ & $1374(43.6)$ \\
\hline Age, median (IQR) & $40(33-46)$ & $40(34-47)$ & $39(32-45)$ \\
\hline Male gender, $n(\%)$ & 2394 (75.9) & $1413(79.3)$ & $981(71.4)$ \\
\hline Caucasian, $n(\%)$ & 2511 (79.6) & $1456(81.8)$ & $1055(76.8)$ \\
\hline Basic education $^{\mathrm{b}}, n(\%)$ & $683(22.0)$ & $337(19.2)$ & $346(25.7)$ \\
\hline \multicolumn{4}{|l|}{ Risk group for HIV infection, $n(\%)$} \\
\hline Men having sex with men & $1568(49.7)$ & $1004(56.4)$ & $564(41.1)$ \\
\hline Heterosexual & $1185(37.6)$ & $606(34.0)$ & $579(42.1)$ \\
\hline IDU & $301(9.5)$ & $123(6.9)$ & $178(13.0)$ \\
\hline Other & $101(3.2)$ & $48(2.7)$ & $53(3.9)$ \\
\hline Past or current IDU ${ }^{\mathrm{C}}, n(\%)$ & $354(11.2)$ & $150(8.4)$ & $204(14.9)$ \\
\hline Psychiatric comorbidity ${ }^{\mathrm{d}}, n(\%)$ & $579(18.4)$ & $320(18.0)$ & 259 (18.9) \\
\hline Living alone, $n(\%)$ & $1301(42.0)$ & $762(43.5)$ & $539(40.1)$ \\
\hline Stable partnership, $n(\%)$ & $1869(60.5)$ & $1058(60.5)$ & $811(60.4)$ \\
\hline \multicolumn{4}{|l|}{ HIV-1 RNA viral load (copies/ml), $n(\%)$} \\
\hline$<50$ & $91(3.0)$ & $58(3.4)$ & $33(2.5)$ \\
\hline $50-399$ & $193(6.4)$ & $110(6.4)$ & $83(6.3)$ \\
\hline$\geq 400$ & $2743(90.6)$ & $1539(90.2)$ & $1204(91.2)$ \\
\hline \multicolumn{4}{|l|}{$\mathrm{CD}^{+}$cell count $(\mu \mathrm{l}), n(\%)$} \\
\hline$<200$ & $930(30.7)$ & $482(28.2)$ & $448(34.0)$ \\
\hline $200-349$ & $1266(41.8)$ & $713(41.7)$ & $553(42.0)$ \\
\hline $350-499$ & $547(18.1)$ & $334(19.5)$ & $213(16.2)$ \\
\hline$\geq 500$ & $286(9.4)$ & $182(10.6)$ & $104(7.9)$ \\
\hline Median (IQR) & $266(178-363)$ & $278(186-375)$ & $255(168-342)$ \\
\hline AIDS, $n(\%)$ & $349(11.1)$ & $190(10.7)$ & 159 (11.6) \\
\hline \multicolumn{4}{|l|}{ ART regimen, $n(\%)$} \\
\hline NNRTI & $1414(44.8)$ & $808(45.4)$ & $606(44.1)$ \\
\hline Protease inhibitor boosted & $67(2.1)$ & $34(1.9)$ & $33(2.4)$ \\
\hline Protease inhibitor nonboosted & $1509(47.8)$ & $850(47.7)$ & $659(48.0)$ \\
\hline Triple nucleoside/other & $165(5.2)$ & $89(5.0)$ & $76(5.5)$ \\
\hline \multicolumn{4}{|l|}{ Regimen backbone, $n(\%)$} \\
\hline $\mathrm{ZDV} / 3 \mathrm{TC}$ & $610(19.5)$ & $251(14.2)$ & $359(26.5)$ \\
\hline $\mathrm{ABC} / 3 \mathrm{TC}$ & $371(11.9)$ & $212(12.0)$ & $159(11.7)$ \\
\hline TDF/3TC & $230(7.4)$ & $85(4.8)$ & $145(10.7)$ \\
\hline TDF/ETC & $1808(57.9)$ & $1164(65.8)$ & $644(47.5)$ \\
\hline Other & $104(3.3)$ & $56(3.2)$ & $48(3.5)$ \\
\hline \multicolumn{4}{|l|}{ Dosing frequency, $n(\%)$} \\
\hline Once daily & $1957(62.1)$ & $1215(68.3)$ & $742(54.0)$ \\
\hline Twice daily & $1190(37.8)$ & $561(31.6)$ & $629(45.8)$ \\
\hline Thrice daily & $4(0.1)$ & $2(0.1)$ & $2(0.2)$ \\
\hline \multicolumn{4}{|l|}{ Number of other HIV patients seen by physician } \\
\hline Median (IQR) & $24(11-69)$ & $26(11-76)$ & $22(11-62)$ \\
\hline \multicolumn{4}{|l|}{ Year of ART initiation, $n(\%)$} \\
\hline $2003-2004$ & $472(15.0)$ & $152(8.5)$ & $320(23.3)$ \\
\hline 2005-2006 & $511(16.2)$ & $217(12.2)$ & $294(21.4)$ \\
\hline $2007-2008$ & $706(22.4)$ & $381(21.4)$ & $325(23.7)$ \\
\hline $2009-2010$ & $881(27.9)$ & $572(32.1)$ & $309(22.5)$ \\
\hline 2011-2012 & $585(18.5)$ & $459(25.8)$ & $126(9.2)$ \\
\hline Time since HIV diagnosis (years), median (IQR) & $1.7(0.2-4.7)$ & $1.4(0.1-4.3)$ & $2.1(0.2-5.1)$ \\
\hline
\end{tabular}

ABC/3TC, abacavir/lamivudine; ART, antiretroviral therapy; ZDV/3TC, zidovudine/lamivudine; ETC, emtricitabine; IDU, injecting drug use; IQR, interquartile range; NNRTI, nonnucleoside reverse transcriptase inhibitors; TDF/3TC, tenofovir/lamivudine .

${ }^{a}$ Nonadherent individuals are those reporting any missed doses throughout the follow-up period.

$\mathrm{b}_{9}$ years of mandatory schooling or less.

${ }^{\mathrm{C}}$ Either IDU transmission group or report of use of injecting drugs.

${ }^{\mathrm{d}}$ Psychiatric morbidity is defined as receiving psychiatric treatment, diagnosis of depression, or taking antidepressants.

dataset included 3155 individuals with 15020 personyears of observation followed for a median 4.7 years (interquartile range: $2.9-6.9$ ) and completing a median of nine questionnaires (interquartile range: $5-15$ ). By the end of follow-up, 1155 (36.6\%) individuals had reported missing one dose, 414 (13.1\%) two doses, 333 (10.6\%) more than two doses of ART, 601 (19.0\%) 95\% or less, and $387(12.3 \%) 90 \%$ or less adherence on at least one occasion. There were $361(11.4 \%)$ individuals lost to follow-up and 104 died (crude mortality rate ratio 6.57,
95\% confidence interval [CI]: 5.42-7.97/1000 personyears). For the outcome of viral failure, an additional 174 individuals were excluded because of the absence of follow-up RNA. Of the eligible 2981 individuals, 220 (7.4\%) individuals were lost to follow-up and 480 (16.1\%) and 1234 (41.4\%) experienced viral failure with a threshold of 500 and 50 copies $/ \mathrm{ml}$, respectively.

Patient characteristics are shown in Table 1. Patients differed in baseline characteristics according to their 
adherence. Individuals who reported missing at least one dose of ART on at least one occasion were more likely to be women, from the heterosexual or injecting drug use transmission group, and initiated ART in earlier years on a b.i.d. regimen without a tenofovir backbone (Table 1).

Results from regression models were consistent across all definitions of nonadherence and viral failure. Compared with no missed doses, the adjusted but unweighted estimate of the risk of viral failure for missing one dose of ART was 1.13 (95\% CI: 0.78-1.64), missing two doses was 2.27 (95\% CI: 1.39-3.73), and missing more than two doses was 5.19 (95\% CI: 3.21-8.41) (Table 2). The corresponding estimates from the MSM were similar with a hazard of 1.15 (95\% CI: 0.79-1.67) for missing one dose, 2.15 (95\% CI: 1.31-3.53) for missing two doses, and 5.21 (95\% CI: 2.96-9.18) for missing more than two doses. When viral failure was defined as HIV-1 RNA more than 50 copies $/ \mathrm{ml}$, the effect of missing only one dose of ART was now significantly associated with viral failure in both unweighted and weighted models (hazard ratio [HR] 1.37, 95\% CI: $1.08-1.74$ and HR 1.49, 95\% CI: $1.17-1.90$, respectively). The MSM models for percentage adherence were comparable with a significant increased risk in viral failure for those taking less than $95 \%$ of medication (90-95\% HR 1.93, 95\% CI: 1.03-3.64; $\leq 90 \%$ HR 6.47, 95\% CI: 3.94-10.63).
In mortality models, the unweighted adjusted mortality HR for missing one dose of ART was 1.31 (95\% CI: $0.62-2.75$ ), for missing two doses 1.06 (95\% CI: $0.25-$ 4.41), and for missing more than two doses 2.89 (95\% CI: 1.13-7.41) compared with no missed doses (Table 2). The mortality HRs from the MSM estimated stronger effects of nonadherence: missing one dose 1.32 (95\% CI: $0.42-4.17)$, missing two doses 1.26 (95\% CI: 0.36-4.44), and missing more than two doses 4.87 (95\% CI: $2.21-$ 10.73). The estimates from MSM model with percentage adherence were similar (Table 2). At baseline, $62.8 \%$ of naive individuals started ART on a q.d. regimen and $37.2 \%$ on a b.i.d. regimen. The number of individuals initiating a q.d. regimen increased from $12.4 \%$ in 2003 to $84.6 \%$ in 2012. We found evidence of an interaction between dosing frequency and missed doses in MSM models; the effect of missed doses on viral failure was different in those missing one to two doses on q.d. and b.i.d. regimens. Compared with missing no doses on either a q.d. or b.i.d. regimen, the effect of missing one to two doses was 1.67 (95\% CI: 1.11-2.50) and $0.99(95 \%$ CI: 0.64-1.54) on a q.d. and b.i.d. regimen, respectively, $(P$ value for interaction $=0.09)$, and missing more than two doses was 5.62 (95\% CI: 2.98-10.62) and $4.05(95 \%$ CI: $1.08-1.46)$ in those on q.d. and b.i.d. regimens $(P$ value for interaction $=0.33$, , respectively. Models with percentage adherence yielded similar results $(90-95 \%$ : q.d. 4.30 [1.62-11.42], b.i.d. 0.69 [0.16-2.94]; <90\%:

Table 2. Models for the effect of nonadherence to antiretroviral therapy on clinical outcomes.

\begin{tabular}{|c|c|c|c|}
\hline & \multicolumn{2}{|c|}{ Viral failure ${ }^{a}$} & \multirow{2}{*}{$\begin{array}{c}\text { Death } \\
\text { HR }(95 \% \mathrm{Cl})\end{array}$} \\
\hline & $\begin{array}{l}>50 \text { copies } / \mathrm{ml} \\
\text { HR }(95 \% \mathrm{Cl})\end{array}$ & $\begin{array}{c}>500 \text { copies/ml } \\
\text { HR }(95 \% \text { Cl })\end{array}$ & \\
\hline \multicolumn{4}{|l|}{ Unweighted estimates ${ }^{b}$} \\
\hline \multicolumn{4}{|l|}{ Missed doses } \\
\hline Missing no doses & Reference & Reference & Reference \\
\hline Missing one dose & $1.37(1.08-1.74)$ & $1.13(0.78-1.64)$ & $1.31(0.62-2.75)$ \\
\hline Missing two doses & $1.86(1.26-2.75)$ & $2.27(1.39-3.73)$ & $1.06(0.25-4.41)$ \\
\hline Missing more than two doses & $3.80(2.49-5.80)$ & $5.19(3.21-8.41)$ & $2.89(1.13-7.41)$ \\
\hline \multicolumn{4}{|l|}{ Percentage adherence } \\
\hline$>95 \%$ & Reference & Reference & Reference \\
\hline $90-95 \%$ & $1.45(0.88-2.40)$ & $2.07(1.10-3.87)$ & $2.56(0.79-8.30)$ \\
\hline$<90 \%$ & $5.00(3.30-7.57)$ & $6.94(4.44-10.86)$ & $2.59(0.85-7.86)$ \\
\hline \multicolumn{4}{|l|}{ Stabilized weighted estimates ${ }^{\mathrm{c}}$} \\
\hline \multicolumn{4}{|l|}{ Missed doses } \\
\hline Missing no doses & Reference & Reference & Reference \\
\hline Missing one dose & $1.49(1.17-1.90)$ & $1.15(0.79-1.67)$ & $1.32(0.42-4.17)$ \\
\hline Missing two doses & $1.96(1.29-2.99)$ & $2.15(1.31-3.53)$ & $1.26(0.36-4.44)$ \\
\hline Missing more than two doses & $4.82(2.60-8.90)$ & $5.21(2.96-9.18)$ & $4.87(2.21-10.73)$ \\
\hline \multicolumn{4}{|l|}{ Percentage adherence } \\
\hline$>95 \%$ & Reference & Reference & Reference \\
\hline $90-95 \%$ & $1.56(0.93-2.63)$ & $1.93(1.03-3.64)$ & $1.85(0.55-6.23)$ \\
\hline$<90 \%$ & $7.79(4.65-13.04)$ & $6.47(3.94-10.63)$ & $4.45(1.99-9.93)$ \\
\hline
\end{tabular}

ART, antiretroviral therapy; $\mathrm{Cl}$, confidence intervals; $\mathrm{HR}$, hazard ratio; IDU, injecting drug use.

${ }^{a}$ Viral failure was defined as the first HIV-1 RNA viral load above the specified threshhold after either achieving viral suppression (viral load $<50$ copies $/ \mathrm{ml}$ ) or being on therapy for longer than 24 weeks.

${ }^{b}$ Models include nonadherence, past nonadherence, and a time-varying intercept as well as the following baseline covariates (age, sex, basic education, Caucasian ethnicity, living alone, having a stable partnership, psychiatric comorbidity, IDU, AIDS, CD4, HIV-1 viral load, class of ART, backbone of regimen, dosing frequency, time period of ART initiation, time since HIV diagnosis, and experience of the treating physician) and timevarying covariates (ART class, dosing frequency, CD4, RNA [mortality model only], and length of time an individual maintained viral suppression [viral failure model only]).

${ }^{\mathrm{c}}$ These models are estimated using MSMs. The time-varying covariates are only included in the model for the weights. 
q.d. 9.01 [4.02-20.21], b.i.d. 4.38 [1.78-10.74]). There were not enough events to explore the interaction in mortality models.

\section{Discussion}

Nonadherence, as assessed by self-report, was found to increase the risk of viral failure and mortality in models adjusting for both time-independent and time-updated variables as well as past adherence. Each additional missed dose was associated with an increase in the risk of viral failure exhibiting a clear dose-response relationship, and missing more than two doses of ART had an approximately five-fold increase in the hazard of viral failure and death. Simplification to q.d. regimens can lead to better adherence but our findings indicate an increased risk of viral failure on q.d. compared with b.i.d. regimens in the presence of nonadherence.

It is difficult to compare our results with previous studies because of different measurement methods and definitions of nonadherence. Our effect estimates are somewhat higher than those reported in earlier studies likely because of proper adjustment for time-dependent confounding (providing the total effect of nonadherence) and less precise because of the lower number of events in our study, particularly deaths $[8,9,16]$.

The present study has several strengths and limitations. Like all observational studies, the effect estimates have a causal interpretation only if there is no unmeasured confounding. The strength of the study was our ability to include many important confounders of the relationship between adherence and clinical outcomes (such as psychosocial factors); however, unmeasured confounders, such as lifestyle and health-seeking behaviors, may bias our estimates. We further strengthened the study by doing extensive sensitivity analyses, which verified the robustness of our estimates and did not change the substantive conclusions from the analysis.

Another limitation of this study is the use of self-reported adherence, which is known to overestimate adherence compared with objective measures $[17,18]$. Investigation of nonadherence by physicians during consultations may lead to an overestimate of adherence because of poor recall or fear to disclose undesirable behavior. Despite this potential bias, this simple adherence question had the sensitivity to detect different effects of missing doses on different viral load thresholds. Self-report is the most likely candidate for widespread use in daily clinical practice because of its many advantages over other measurement methods, such as low cost and ease of administration.

The SHCS is a long-standing and well described cohort that collects a variety of information longitudinally in a diverse population, including understudied groups such as heterosexuals, drug users, and women, increasing the generalizability of the results. Regardless, additional validation of these findings in other patient populations and cultural settings is warranted.

Over an average follow-up of 5 years, one out of 10 patients reported having missed more than two doses of ART during the last 4 weeks. Regular investigation of adherence at each clinic visit is therefore crucial and reports of nonadherence should alert physicians to not only provide adherence support but to explore potential underlying reasons for the lack of adherence. This opportunity for early and appropriate intervention could lead to a reduction in drug failure and resistance. The sensitivity of this simple adherence question to predict poor clinical outcomes has wide implications and should be considered for use in resource-limited settings where viral load often cannot be measured.

\section{Acknowledgements}

The authors thank the patients participating in the SHCS for their commitment, the study physicians and nurses for excellent patient care, and the SHCS laboratories for providing high quality data and the data center for state of the art data management.

The members of the Swiss HIV Cohort Study are Aubert V, Battegay M, Bernasconi E, Böni J, Bucher HC, Burton-Jeangros C, Calmy A, Cavassini M, Dollenmaier G, Egger M, Elzi L, Fehr J, Fellay J, Furrer H (Chairman of the Clinical and Laboratory Committee), Fux CA, Gorgievski M, Günthard H (President of the SHCS), Haerry D (deputy of 'Positive Council'), Hasse B, Hirsch HH, Hoffmann M, Hösli I, Kahlert C, Kaiser L, Keiser O, Klimkait T, Kouyos R, Kovari H, Ledergerber B, Martinetti G, Martinez de Tejada B, Metzner K, Müller N, Nadal D, Nicca D, Pantaleo G, Rauch A (Chairman of the Scientific Board), Regenass S, Rickenbach M (Head of Data Center), Rudin C (Chairman of the Mother \& Child Substudy), Schöni-Affolter F, Schmid P, Schüpbach J, Speck R, Tarr P, Trkola A, Vernazza P, Weber R, Yerly S.

Authors' contributions: T.R.G. and H.C.B. designed the experiment. M.-P.S., D.N., H.F., H.F.G., E.B., A.C., M.B., and H.C.B. recruited patients for the study. M.R. is responsible for data quality and extraction. T.R.G. analyzed data and performed statistical analysis. T.R.G., J.A.C.S., and H.C.B. interpreted the results. All authors contributed to the writing of the article.

Source of funding: This study has been financed within the framework of the Swiss HIV Cohort Study, supported by the Swiss National Science Foundation 
(grant \# 148522) and by SHCS project 608. J.A.C.S. was funded by the UK Medical Research Council [grant number MR/J002380/1], the Department for International Development (D.F.I.D.) and UK National Institute for Health Research Senior Investigator award NF-SI-0611-10168. The Basel Institute for Clinical Epidemiology and Biostatistics is supported by grants from Santésuisse and the Gottfried and Julia BangerterRhyner-Foundation.

\section{Conflicts of interest}

There are no conflicts of interest.

\section{References}

1. Sterne JA, Hernan MA, Ledergerber B, Tilling K, Weber R, Sendi $P$, et al. Long-term effectiveness of potent antiretroviral therapy in preventing AIDS and death: a prospective cohort study. Lancet 2005; 366:378-384.

2. Mocroft A, Ledergerber B, Katlama C, Kirk O, Reiss $P$, d'Arminio MA, et al. Decline in the AIDS and death rates in the EuroSIDA study: an observational study. Lancet 2003; 362:22-29.

3. Lundgren JD, Mocroft A. The impact of antiretroviral therapy on AIDS and survival. J HIV Ther 2006; 11:36-38.

4. Glass TR, De GS, Hirschel B, Battegay M, Furrer H, Covassini M, et al. Self-reported nonadherence to antiretroviral therapy repeatedly assessed by two questions predicts treatment failure in virologically suppressed patients. Antivir Ther 2008; 13:77-85.

5. Bangsberg DR, Acosta EP, Gupta R, Guzman D, Riley ED, Harrigan PR, et al. Adherence-resistance relationships for protease and nonnucleoside reverse transcriptase inhibitors explained by virological fitness. AIDS 2006; 20: 223-231.

6. Lima VD, Harrigan R, Bangsberg DR, Hogg RS, Gross R, Yip B, et al. The combined effect of modern highly active antiretroviral therapy regimens and adherence on mortality over time. J Acquir Immune Defic Syndr 2009; 50:529-536.
7. von Wyl V, Klimkait T, Yerly S, Nicca D, Furrer H, Cavassini M, et al. Adherence as a predictor of the development of classspecific resistance mutations: the Swiss HIV Cohort Study. PLoS One 2013; 8:e77691.

8. Lima VD, Hogg RS, Harrigan PR, Moore D, Yip B, Wood E, et al. Continued improvement in survival among HIV-infected individuals with newer forms of highly active antiretroviral therapy. AIDS 2007; 21:685-692.

9. Villes V, Spire B, Lewden C, Perronne C, Besnier JM, Garre M, et al. The effect of depressive symptoms at ART initiation on HIV clinical progression and mortality: implications in clinical practice. Antivir Ther 2007; 12:1067-1074.

10. Garcia de Olalla P, Knobel H, Carmona A, Guelar A, LopezColomes JL, Cayla JA. Impact of adherence and highly active antiretroviral therapy on survival in HIV-infected patients. J Acquir Immune Defic Syndr 2002; 30:105-110.

11. Hernan MA, Brumback B, Robins JM. Marginal structural models to estimate the causal effect of zidovudine on the survival of HIV-positive men. Epidemiology 2000; 11:561570.

12. Hernan MA. A definition of causal effect for epidemiological research. J Epidemiol Community Health 2004; 58:265-271.

13. Lima VD, Harrigan R, Murray M, Moore DM, Wood E, Hogg RS, et al. Differential impact of adherence on long-term treatment response among naive HIV-infected individuals. AIDS 2008; 22:2371-2380.

14. Glass TR, De Geest $S$, Weber R, Vernazza PL, Rickenbach M, Furrer $\mathrm{H}$, et al. Correlates of self-reported nonadherence to antiretroviral therapy in HIV-infected patients: the Swiss HIV Cohort Study. J Acquir Immune Defic Syndr 2006; 41:385-392.

15. Schoeni-Affolter $F$, Ledergerber $B$, Rickenbach $M$, Rudin $C$, Gunthard HF, Telenti A, et al. Cohort profile: the Swiss HIV Cohort study. Int J Epidemiol 2010; 39:1179-1189.

16. Hogg RS, Heath K, Bangsberg D, Yip B, Press N, O'Shaughnessy $M V$, et al. Intermittent use of triple-combination therapy is predictive of mortality at baseline and after 1 year of follow-up. AIDS 2002; 16:1051-1058.

17. Berg KM, Arnsten JH. Practical and conceptual challenges in measuring antiretroviral adherence. I Acquir Immune Defic Syndr 2006; 43 (Suppl 1):S79-S87.

18. Glass TR, Cavassini M. Asking about adherence: from flipping the coin to strong evidence. Swiss Medical Weekly 2014; 144:w14016. 